DOI: https://doi.org/10.30837/MMP.2021.182

\title{
SIMULATION MODEL OF TECHNOLOGICAL PROCESS OF RADIO ELECTRONICS PRODUCTS ASSEMBLY ON THE BASIS OF FLEXIBLE SWITCHING STRUCTURES
}

\author{
Nevlyudov I., Starodubcev N., Vlasenkov D., Andrusevich A.
}

Modern technologies of assembling radio electronic products based on aluminumpolyamide flexible switching structures (FSS) are a complex set of technological problems related to the development of new design technological principles for the design of technological processes (TP), ensuring their reliability, controllability [1-4]. The problem of providing the necessary levels of quality and technical excellence of manufactured electronic products aims at the use of effective methods of assembling elements of functional electronics on flexible boards-bases [5-7], at improving the methods of modeling TP assembly, which must provide high informativeness and reliability [8-11]. It is necessary to develop an effective mathematical support for modeling the functioning and evaluation of the reliability of TP assembly and installation of FSS-based electronic products. A simulation model of the technological process of assembling a type-forming electronic product on an aluminum-polyimide basis with the use of a mathematical apparatus, special instrumental and software tools (GPSS World) of the theory of mass service is presented, which made it possible to obtain a mathematical model of TP assembly of radio electronic products to determine the percentage of the output of suitable electronic products manufactured on the assembly process line

\section{INTRODUCTION}

During TP modeling of ET modules using mathematical apparatus and special tools (GPSS World), mass-service theory in general solves two problems [12-15]. Let's call them straight and reverse.

A direct problem is to determine the estimation of the mathematical expectation of any indicator of a modeled technological system at a given time of its functioning.

The inverse of the problem is to determine the estimate of the mathematical expectation of the time of operation of the system, during which any of its indicators reaches a given value. 
Solving these problems, especially the inverse problem, has its own peculiarities. Let us consider these features further on the example of modeling TP assembly of a sample-representative of ET modules on an aluminum-polyimide basis, the main technical characteristics of which are given in [16].

\section{FORMULATION OF THE PROBLEM}

One of the main structural elements of the analyzed ET modules are crystals of integrated circuits (IS) or hybrid microsborders mounted on the surface of a flexible aluminum-polyimide carrier board [17].

Assembly of the electronic module begins after a random time. Operations are preceded by the preparation of a constructive element (CE), which includes input control. The duration of preparation depends on the degree of preparation for the assembly of the initial element base, from the elements of which the final product will be assembled. All different types of constructive elements of electronic product (CE) - $n_{1}$ (hybrid microscaling, three silicon sensors (No. 1, No. 2, No. 3), connecting cables No. 1 and No. 2). The frequency of appearance of different CE and the average values of their preparation time (in minutes) are specified in Table 1 of discrete distribution.

Table 1

The frequency of $\mathrm{CE}$ and the average time of their preparation

\begin{tabular}{|c|c|c|c|c|c|c|}
\hline Structural element & $\begin{array}{c}\text { Connecting } \\
\text { cable No. 1 }\end{array}$ & $\begin{array}{c}\text { Connecting } \\
\text { cable No. 2 }\end{array}$ & $\begin{array}{c}\text { Sensor } \\
\text { No. 1 }\end{array}$ & $\begin{array}{c}\text { Sensor } \\
\text { No. 2 }\end{array}$ & $\begin{array}{c}\text { Sensor } \\
\text { No. 3 }\end{array}$ & $\begin{array}{c}\text { Micro- } \\
\text { composition }\end{array}$ \\
\hline Frequency, min & 0,05 & 0,13 & 0,16 & 0,15 & 0,22 & 0,29 \\
\hline $\begin{array}{c}\text { Average time of } \\
\text { preparation of CE, } \\
\text { min }\end{array}$ & 10 & 14 & 21 & 25 & 22 & 28 \\
\hline
\end{tabular}

For the assembly of ET modules, operations are sequentially performed (placing CEs on the mounting table, positioning CEs relative to each other, installing CE using ultrasound micro-welding) with medium time $T_{1}, T_{2}$ and $T_{3}$, accordingly. After each operation during the time $T_{k 1}, T_{k 2}, \ldots, T_{k n}$ interoperative control is 
performed. Time of operations and control - random. Control does not pass (discarded) $q_{1}, q_{2}, \ldots, q_{n}$ products respectively.

Defective products are sent to the point of final control (acceptance control) and are inspected. As a result, out of the total number of products that have not passed the control, $q_{n+1} \%$ are missing, and those $1-q_{n+1} \%$ remaining ET modules are subject to re-execution of those operations after which they did not pass the control. If the electronic product is not controlled for a second time, it is permanently rejected.

The initial data for building the model are as follows:

$n_{1}=6$; Exponential TP = Exponential 30; q1 = $12 \%$, q2 = $15 \%$;

$\mathrm{n}=6$; Exponential $\left(T_{1}\right)=$ Exponential $(30) ; q_{3}=10 \%, q_{4}=80 \%$;

Exponential (T2) = Exponential (25); Exponential (T3) = Exponential (35);

Exponential $(T k 1)=$ Exponential (4); Exponential $(T k 2)=$ Exponential (5);

Exponential $(\mathrm{Tk} 3)=$ Exponential $(15) ;$ Exponential $(\mathrm{T \kappa})=$ Exponential (8).

In the end, we obtain a simulation mathematical model of TP assembly of products - ET modules - to determine the estimate of the mathematical expectation of the number of products manufactured on the assembly line for 8 hours.

The model will allow to perform a generalized assessment of the reliability and stability of TP - to determine the relative number of suitable and rejected electronic modules, the average manufacturing time of one product.

The results of the simulation are obtained with accuracy $\varepsilon=0,01$ and confidence probability $\alpha=0,99[12,13]$.

The process of assembling ET modules is a process that takes place in a multiphase open queuing system with waiting (fig. 1). There are also signs of a closed system - the flow of defects for reassembly, after the elimination of defects.

We will present that preparation of CE and operations of assembly 1, 2 and 3 (placement of CE on an assembly table, positioning of CE concerning each other, installation of CE by means of UZ-microwelding) are carried out on installations single-channel devices (SCD) 1, 2, 3 and 4. respectively (on technological installations of control, placement and positioning, installation of UZ- 
microwelding). The point of final control (acceptance) can also be represented as SCD. The GPSS tools needed to simulate them are shown in fig. 1.

CE preparation time and transaction execution time are set in minutes. Let's take 1-unit mod. time $=1 \mathrm{~min}$.

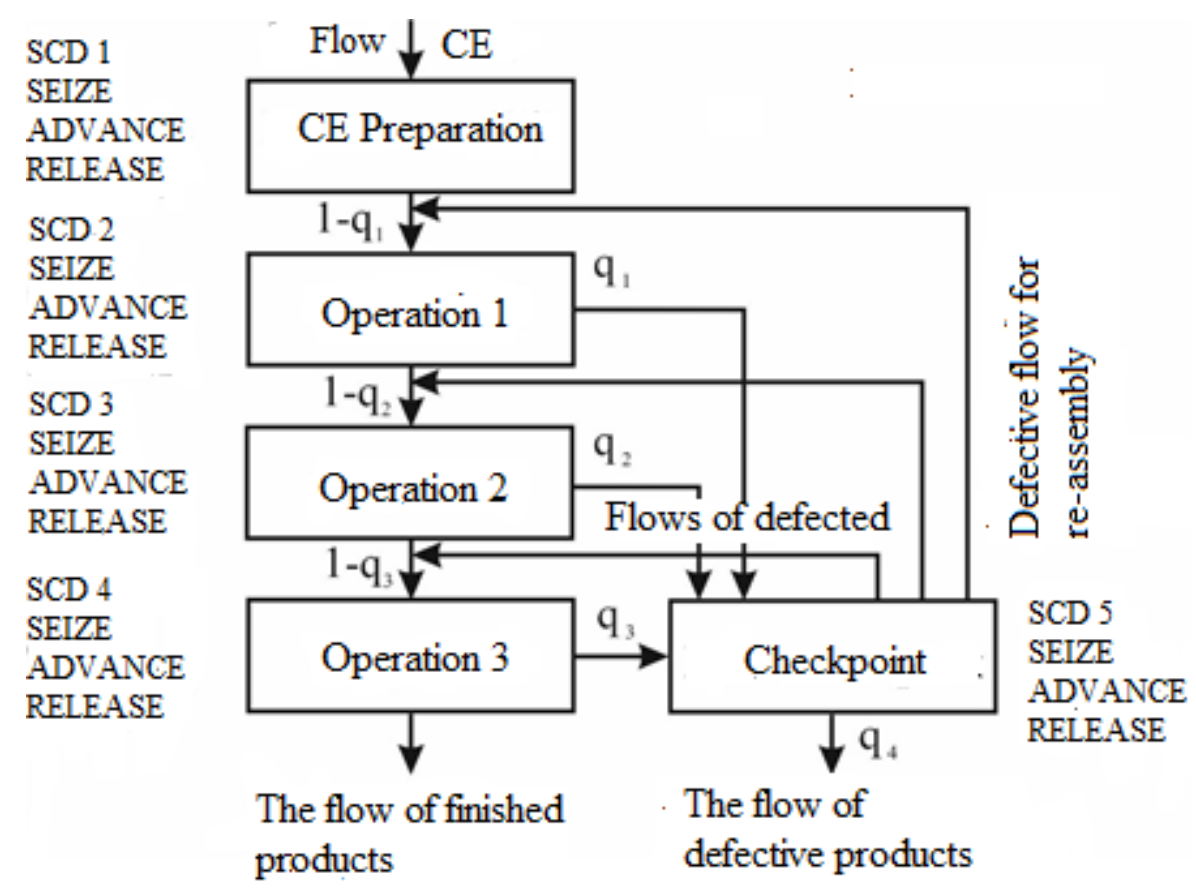

Fig. 1. TP assembly of ET modules as a system of mass maintenance

Let's calculate the number of runs that need to be performed in each observation, i.e. carry out the so-called tactical planning of the experiment. Let the simulation results (query processing probability) be obtained with confidence probability $\alpha=0,95$ and accuracy $\varepsilon=0,01$. We will perform calculations for the worst case, i.e. in probability $\varepsilon=0,01$, because before the experiment $p$ is unknown:

$$
N=t_{\alpha}^{2} \cdot \frac{p \cdot(1-p)}{\varepsilon^{2}}=2,58^{2} \cdot \frac{0,5 \cdot(1-0,5)}{0,01^{2}} \approx 16642
$$

\section{CONSTRUCTING A DIRECT PROBLEM MODEL}

The direct problem model is given below. 
; Model of the process of manufacturing parts of ET modules. Direct problem

; Setting of the output data

Timemod EQU 480; Simulation time, 1-unit mod. time = 1 min

; $\quad$ Average time

Tn_EQU 35 ; between CE receipts

T1 EQU 30 ; execution of the 1st operation, min

T2 EQU 25 ; execution of the 2nd operation, min

T3 EQU 35 ; execution of the 3rd operation, min

Tkl EQU $4 \quad$; control after the 1st operation, min

Tk2 EQU 5 ; control after the 2nd operation, min

Tk3 EQU 15 ; control after the 3rd operation, min

Tk EQU 8 ; final control, min

ql_EQU .12 ; share of defects after the 1st operation

q2 EQU .15 ; share of defects after the 2nd operation

q3 EQU .10 ; share of defects after the 3rd operation

q4_EQU .80 ; share of final defects

; Description of the time function of preparation of blanks

Pod FUNCTION RN10, D6

$.05,10 / .18,14 / .34,21 / .56,22 / .85,28 / 1,25$

; The segment of simulation of assembly of ET modules

GENERATE (Exponential(23, 0, Tn_ )) ; ERE Source

; ERE preparation for ET modules

QUEUE Pod ; Stand in queue

SEIZE Pod $\quad$; Start CE preparation

DEPART Pod ; Leave queue

ADVANCE (Exponential (34, 0, FN\$Pod) ) ; Preparation

RELEASE Pod ; Complete CE preparation

; Simulation of the 1st operation

Dcount ASSIGN 1,1 ; Code 1 - passes for the first time

ASSIGN 2,1 ; Code 1 in P2-sign of the 1st operation 
Operl QUEUE P2 ; Stand in queue

SEIZE Konveer1 ; Start the 1st operation

DEPART P2 ; Leave the queue

ADVANCE (Exponential(23,0,Tl) ) ; 1st operation

RELEASE Konveerl ; End the 1st operation

ADVANCE (Exponential(23,0,Tkl)) ～; Control of the 1st operation

TRANSFER q 1_,Sboi ; Defect to the checkpoint

; Simulation of the 2nd operation

ASSIGN 2,2

; Code 2 in P2-sign of the 2nd operation

Opeг2 QUEUE P2

; Stand in queue

SEIZE Konveer2

; Start the 2nd operation

DEPART P2

; Leave the queue

ADVANCE (Exponential(23,0,T2)) ; the 2nd operation

RELEASE Konveer2 ; End the 2nd operation

ADVANCE (Exponential(23,0,Tk2)) ; Control the 2nd operation

TRANSFER q2_,, Sboi ; Defect to the checkpoint

; Simulation of the 3rd operation

ASSIGN 2,3 ; Code 3 in P2-sign of the 3rd operation

Орегз QUEUE P2 ; Stand in queue

SEIZE Konveer3 ; Start the 3rd operation

DEPART P2 ; Leave the queue

ADVANCE (Exponential $(23,0, T 3)$ ) ; the 3rd operation

RELEASE Konveer3 ; End the 3rd operation

ADVANCE (Exponential(23,0,Tk3)) ; Control of the 3rd operation

TRANSFER q3_,, Sboi ; Defect to the checkpoint

Endoperl TERMINATE ; Counting of finished products

; Segment of simulation of checkpoint operation

Sboi TEST E P1, 1, Endoper ; If the second time, to the final defect

QUEUE Kont ; To the queue at the checkpoint

SEIZE Kontr ; Take the checkpoint 
DEPART Kont ; Leave

ADVANCE (Exponential(23,0,Tk)) ; Final control

RELEASE Kontr ; Release the checkpoint

TRANSFER q4_, Endoper _ ; To the final defect

ASSIGN 1,2 ; Code 2 in the P1 product will go a second time

Metl TRANSFER,(Metl+P2)

TRANSFER,Operl ; Repeatedly for the 1st operation

TRANSFER,Oper2 ～; Repeatedly for the 2nd operation

TRANSFER,Орегз ; Repeatedly for the 3rd operation

Endoper TERMINATE ; Defect calculation

; Segment of simulation time task and calculations of simulation results

GENERATE TimeMod ; Simulation time

TEST L X\$Prog,Tgl,Metl 1 ; If the condition is met, then

SAVEVALUE Prog,Tgl $\quad$; X $\$$ Prog=Tgl the contents of the completion counter

Met 11 TEST E Tgl,1,Metl2 ; If the counter content is 1, then calculation of simulation results

SAVEVALUE Nizd,(N\$Endoperl/X\$Prog) ; Number of finished products, pcs.

SAVEVALUE Brak, m(N\$Endoper/X\$Prog); Number of rejected products, pCs.

SAVEVALUE Doljabrak, $(\mathrm{X} \$ \mathrm{Brak} /(\mathrm{X} \$ \mathrm{Brak}+\mathrm{X} \$ \mathrm{Nizd}))$; The total share of defect

SAVEVALUE Doljaizd,(X\$Nizd/(X\$Brak+X\$Nizd)) ; Share of finished products

SAVEVALUE Nizd,(INT(X\$Nizd)) ; Number of finished products (whole), pcs.

SAVEVALUE Brak,(INT(X\$Brak)) ; Number of rejected products (whole), pCs. 
SAVEVALUE Sizd,((Acl-x\$AC2)/N\$Endoperl); The average assembly time of one product, min

SAVEVALUE AC2,AC1

\section{Met 12 TERMINATE 1}

START 1000,NP

RESET

START 16641
; Number of previous runs

; Reset statistics

; Number of main runs

Let's consider some features of the above software implementation of the model TP assembly of ET modules.

The discrete Pod function is used to set the initial data - CE preparation time. This allows you to reduce the program compared to if you use the EQU command $[12,13]$. In addition, the event part of the model is simplified, because in the simulation unit of the ERE preparation

ADVANCE(Exponential(23,0,FN\$Pod)); Imitation of ERE preparation

it is enough to specify only the reference FN\$Pod to the function.

Codes 1 and 2, written in parameter 1 of the transaction, serve as signs of failure of the product to control the first and second time, respectively. Sign 2 is the basis for sending the product to waste and excluding it from the assembly process.

To count the number of finished and rejected products, the labels EndOper1 and EndOper are introduced, respectively. Since these numbers are accumulated for all runs, to obtain the average values, they are divided by the number of runs X\$Prog, rounded to the whole procedure INT and entered in the stored slots, Ilzd and Brak, respectively. Next, these average values are used to calculate the relative shares of finished Doljalzd and rejected Doljabrak products

The average Sizd assembly time of one product is defined as the ratio of the absolute model time AC1 to the number of collected products for all runs, i.e. to N\$Endope1. 
This would be correct if there were no previous runs of the model. The model time of these runs should not be taken into account when determining the average assembly time of the product.

It should be remembered after the previous runs.

A command is entered for this

SAVEVALUE AC2, AC1 ; Time of previous runs

After the main runs, calculations are performed:

SAVEVALUE Sizd, ((AC1-X\$C2)/N\$Endoper1); The average manufacturing time of one part, min.

With the help of the GPSS World software package, the process of assembling the ET-representative module was modeled. A snippet of the report is given below.

$\begin{array}{lcc}\text { SAVEVALUE } & \text { RETRY } & \text { VALUE } \\ \text { PROG } & 0 & 16641.000 \\ \text { NIZD } & 0 & 9.000 \\ \text { BRAK } & 0 & 3.000 \\ \text { DOLJABRAK } & 0 & 0.279 \\ \text { DOLJAIZD } & 0 & 0.721 \\ \text { SIZD } & 0 & 48.559\end{array}$

As a result of solving the direct problem of simulation, we obtain that in 8 hours Nizd 9 electronic modules will be assembled on the production line, the relative share of finished products will be Doljaizd $=0.721$, and the average assembly time of one product Sizd $=48.559$ minutes. This will reject Brak $=3$ products, the relative share of which will be Doljabrak $=0.2279$. 


\section{BUILDING A MODEL OF THE INVERSE PROBLEM}

The purpose of the inverse simulation problem is to determine the average time to manufacture a certain number of ET modules. To test the efficiency of the model, take the number of products obtained as a result of solving a direct problem, i.e. $I z d=9$. The model for solving the inverse problem is given below .

; Model of product assembly process. The inverse problem

; Simulation of the 3rd operation

ASSIGN 2,3

Орегз QUEUE P2

SEIZE Konveer3

DEPART P2
; Code 3 in P2-sign of the 3rd operation

; Stand in queue

; Start the 3rd operation

; Leave the queue

ADVANCE (Exponential $(23,0, \mathrm{~T} 3))$

; 3rd operation

RELEASE Konveer3

; End the 3rd operation

ADVANCE (Exponential (23,0,T3)); Control of the 3rd operation

TRANSFER q3_,Sboi

TRANSFER,Met2
; Defect to a checkpoint

; Finished electronic products

; Segment of simulation of checkpoint operation

Sboi QUEUE Kont

SEIZE Kontr

DEPART Kont
; To the queue at the checkpoint

; Take the checkpoint

; Leave the queue at the checkpoint

ADVANCE (Exponential(23,0,Tk)) ; Final control

RELEASE Kontr

; Release the checkpoint

TRANSFER q4_,Endoper

TEST E Pl,l,Endoper

; To the final defect

ASSIGN 1,2

; If the second time, the final defect

; Code 2 in the P1 product will go a second time

Metl TRANSFER,(Metl+P2)

TRANSFER,Operl

; Repeatedly for the 1st operation

TRANSFER,Oper2 
TRANSFER,Орегз ; Repeatedly for the 3rd operation

Endoper TERMINATE ; Defect counting

; Segment of completion of modeling and calculation of results

Met2 TEST L X\$Prog,Tgl,Met3 ; If the condition is met, then

SAVEVALUE Prog,Tgl ; X $\quad$ \$Prog=Tgl completion counter

Met3 SAVEVALUE Nizd+, ; Counting the number of finished products

TEST E X\$Nizd,Izd,Terl ; If Izd details are ready, fix one run

TEST E Tgl,1,Met4 ; If the counter counts 1, then the calculations of the simulation results

SAVEVALUE Brak,(N\$Endoper/X\$Prog) ; Number of rejected products, pcs.

SAVEVALUE Doljabrak,(X\$Brak/(X\$Brak+Izd) ; The total part of the defects

SAVEVALUE Doljaizd,(Izd/(X\$Brak+Izd)) ; The share of finished products

SAVEVALUE Brak,(INT(X\$Brak)) ; Number of rejected products (whole), pcs.

SAVEVALUE Tizd,(((Acl-x\$AC2)/X\$Prog)/60) ; Average time of production of Izd products, hours

SAVEVALUE Sizd,((X\$Tizd/Izd)\#60) ; The average production time of one item, min

SAVEVALUE AC2,AC1 ; Time of previous runs

SAVEVALUE X\$Prog,0 ; Resetting the jack X\$Prog

Met4 SAVEVALUE Nizd,0 ; Resetting X\$Nizd

TERMINATE 1 ; From the completion counter minus 1

Terl TERMINATE ; Output of auxiliary transactions

START 1000,NP ; $\quad$; The number of previous runs of the model

RESET ; Resetting statistics

START 16641 ; The number of main runs of the model 
In the given software implementation of the TP model for solving the inverse problem, in order to reduce it, the text is excluded to the part that simulates the execution of the 3rd operation, because it is the same as in the program model for solving the direct problem. The only difference is that a command should be added to the source data to specify the number of products to be manufactured.:

Izd. EQU 9 ; Number of products to be manufactured

Rounding to the whole number of rejected products is carried out after calculating the share of finished and rejected products. If not, the calculation of the shares will be incorrect.

In the program of the model of the direct problem suitable products after execution of the 3rd operations were counted. Transactions imitating them were destroyed:

Endoper1 TERMINATE $\quad$; Counting of finished products

In the program of the inverse problem model, suitable products must be sent to the segment of the organization to complete the simulation. For this purpose, the above block of calculation of finished products is replaced:

TRANSFER,Met2 ; Finished products

The simulation segment of the control point remains unchanged. Let's focus on the segment of modeling completion and calculation of results.

The stored Nizd slot is used to count the current number of manufactured products. As soon as the condition X\$Nizd = Izd is fulfilled, one run of the model is fixed. Izd is a user variable that specifies the number of products whose preparation time needs to be determined. 
The model time of previous runs should not be taken into account when calculating the average manufacturing time of 9 ET modules. Therefore, it is stored in the stored slot, $\mathrm{X} \$ \mathrm{AC} 2$, and during the calculations is subtracted from $\mathrm{AC} 1$ :

SAVE VALUE Tizd,(((Acl-x\$AC2)/X\$Prog)/60) ; Average assembly time for Izd products, hours

Using the GPSS World software package, the modeling process of the ET module was modeled. A snippet of the report is below:

\begin{tabular}{lcc}
\multicolumn{1}{c}{ SAVEVALUE } & RETRY & VALUE \\
PROG & 0 & 16641.000 \\
BRAK & 0 & 3.000 \\
DOLJABRAK & 0 & 0.279 \\
DOLJAIZD & 0 & 0.721 \\
TIZD & 0 & 7.266 \\
SIZD & 0 & 48.559
\end{tabular}

As a result of solving the inverse problem, we obtain that 9 products, the relative share of which will be Doljaizd $=0.721$, will be made in Tizd $=7.266$ hours, and the average assembly time of one product Sizd $=48.443$ minutes. This will reject $B r a k=3$ products, the relative share of which will be Doljabrak $=0.279$.

\section{ANALYSIS OF TP MODELING RESULTS FOR ET MODULES ASSEMBLING}

Thus, in the framework of this section, a stochastic model of TP assembly of ET modules was developed with the help of the mathematical apparatus of queuing theory, and simulation modeling of TP assembly operation was carried out with the help of GPSS World software.

16641 runs of the model were performed in the GPSS World system. The initial values of the random number generator were set sequentially as follows: 23, 731, 9722, 72685 (table 2). 
In addition, the simulation was performed first during the operation of the assembly line 8 hours (480 $\mathrm{min})$, and then 48 hours (2880 $\mathrm{min}$ ).

The obtained indicators are given in table 2.

Such an indicator as the relative share of finished parts (1 and 3) differs by $0 \ldots 0.001$, and the average manufacturing time of one part (2 and 4) by $0.075 \ldots 0.123$ minutes.

If the initial number of the random number generator changes, these indicators do not change significantly.

Table 2

Performance indicators of TP assembly of ET modules

\begin{tabular}{|l|c|c|c|c|}
\hline \multirow{2}{*}{ Indicators } & \multicolumn{4}{|c|}{ Initial numbers of the random number generator } \\
\cline { 2 - 5 } & $\mathbf{2 3}$ & $\mathbf{7 3 1}$ & $\mathbf{9 7 2 2}$ & $\mathbf{7 2 6 8 5}$ \\
\hline \multicolumn{5}{|c|}{ Operating time of the assembly line 8 hours (480 min) } \\
\hline finProduct & 9,885 & 9,909 & 9,884 & 9,905 \\
\hline shareFinProd & 0,721 & 0,721 & 0,721 & 0,722 \\
\hline defectProduct & 3,821 & 3,833 & 3,823 & 3,821 \\
\hline shareDefProd & 0,279 & 0,279 & 0,279 & 0,278 \\
\hline avTPrepProd & 48,559 & 48,440 & 48,563 & 48,460 \\
\hline$\Delta$ shareFinProd & \multicolumn{5}{|c|}{$\Delta_{1}=|0,721-0,722|=0,001$} \\
\hline$\Delta$ avTPrepProd & $\Delta_{2}=|48,563-48,440|=0,123$ \\
\hline \multicolumn{5}{|c|}{ Operating time of the assembly line 48 hours $(2880$ min) } \\
\hline finProduct & 59,496 & 59,405 & 59,432 & 59,496 \\
\hline shareFinProd & 0,722 & 0,722 & 0,722 & 0,722 \\
\hline defectProduct & 22,890 & 22,855 & 22,850 & 22,890 \\
\hline shareDefProd & 0,278 & 0,278 & 0,278 & 0,278 \\
\hline avTPrepProd & 48,406 & 48,481 & 48,459 & 48,406 \\
\hline$\Delta$ shareFinProd & \multicolumn{5}{|c|}{$\Delta_{3}=|0,722-0,722|=0$} \\
\hline$\Delta$ avTPrepProd & $\Delta_{4}=|48,481-48,406|=0,075$ \\
\hline
\end{tabular}

\section{CONCLUSIONS}

Thus, for the operating time of the assembly line 8 hours (480 $\mathrm{min}$ ) the relative proportion of finished parts ( 1 and 3 ) differs by 0.001 , and the average manufacturing 
time of one part ( 2 and 4) - by $0.123 \mathrm{~min}$, for the operating time of the assembly line 48 hours (2880 $\mathrm{min}$ ) the relative proportion of finished parts ( 1 and 3 ) is the same (the difference is 0 ), and the average manufacturing time of one part (2 and 4) differs by $0.075 \mathrm{~min}$. The obtained indicators indicate the adequacy of the simulation results in the GPSS World system

The offered mathematical model of functioning of TP of assembly of the electronic module-type representative allows to carry out forecasting of reliability and stability of TP - to define relative quantity of suitable products, average time of manufacturing of one product, average quantity of ET modules made for change.

\section{References}

1. Technological quality assurance of flexible switching structures: monograph / I.Sh. Nevlyudov, I.V. Botsman, V.V. Nevlyudova, E.A. Razumov-Fryziuk. - Kriviy Rig: KK NAU, 2018. -256 p.

2. Nevlyudova V.V. Application of flexible printed structures in products of modern electronic technology / V.V. Nevlyudova, E.A. Razumov-Fryziuk // Radioelectronics and Informatics. 2016 - No.3. p. 7-12.

3. Demska N.P. Flexible switching structures: analysis of technologies and areas of application / N.P. Demska // Instrumentation technology. - 2019, No.1. p. 38-42

4. Nevlyudov I.Sh. The latest design and technological solutions for ultralight detector modules for physical experiments / I.Sh. Nevlyudov, V.M. Borshchov, I. T. Timchuk, M.A. Protsenko, N.P. Demska // The current state of research and technology in industry. - 2018, No. 3 (5) - p. 67-77.

5. Andrusevich A.A. Investigation of the process of forming micro-joints by ultrasonic welding in the manufacture of flexible printed circuit boards / A.A. Andrusevich, N.G. Starodubcev, V.V. Nevlyudova, D.P. Vlasenkov // VII International Science and Technology Conference "Physical Processes and Fields of Technical and Biological Objects": materials of the conference. Kremenchuk: KNU im. Mikhail Ostrogradsky, 2018. - p. 134-136.

6. Nevlyudov I. Investigation of factors influencing the process of forming welded microjoints in electronic modules / I. Nevlyudov, M. Omarov, I. Botsman, N. Demska, V. Nevlyudova., N. Starodubcev // ICONAT-2019, Eskişehir Technical University Journal of Science and Technology A - Applied Sciences and Engineering, Vol. 20. p. 181-187. 
7. Demska N.P. Technology of electrical interconnections of electronic equipment modules: dis. ... k. t. n. / N.P. Demska. - Kharkiv, 2020. - 200 p.

8. Botsman I.V. Forecasting the parameters of flexible structures in solving the problem of ensuring their quality / I.V. Botsman, N.P. Demska, V.V. Nevlyudova, S.P. Novoselov // Instrumentation technology. - 2017, - No. 1. - p. 42-47.

9. Botsman I.V. Development of models of flexible switching structures for the automation of their design / I.V. Botsman, B.A. Stepanenko, V.V. Nevlyudova // XV International scientific conference "Physical processes and fields of technical and biological objects": conference proceedings. - Kremenchuk: KNU im. Mikhail Ostrogradsky, 2016. - p. 120-121.

10. Nevlyudov I.Sh. Development of a simulation model of the technological process of assembling radio electronic devices based on flexible switching structures / I.Sh. Nevlyudov, A.A. Andrusevich, N.P. Demska // XXVII International conference "New leading technologies in machine building”. Koblevo-Kharkov, 2017. p. 81.

11. Nevlyudov I.Sh. Development of a parametric model of flexible switching structures for the study of mechanical effects on them / I.Sh. Nevlyudov, S.P. Novoselov, N.P. Demska, I.V. Botsman // VII International scientific conference "Physical processes and fields of technical and biological objects": conference proceedings. - Kremenchuk: KNU im. Mikhail Ostrogradsky p. - C. 141-143.

12. Kudryavtsev E.M. GPSS World. Basics of simulation of various systems / E.M. Kudryavtsev. - M .: DMK Press, 2004. - 320 p.

13. Boyev V.D. Investigation of the adequacy of GPSS World and AnyLogic in modeling discreteevent processes: monograph / V.D. Boyev. - SPb.: VAS, 2011. - 404 p.

14. Nevlyudov I. Sh. An Application of the Finite Element Method for Flexible PCB Components Tense-Deformed State Simulation / I. Sh. Nevlyudov, I. S. Khatnyuk // Materials of the XI International Conference "Modem problems of radio engineering, telecommunications and computer science”. Collection of the scientific papers. Lviv - Slavske, 2012. - P. 537.

15. Kryukov A.Yu. Mathematical modeling of processes in mechanical engineering: monograph / A.Yu. Kryukov, B.F. Potapov. - Perm': PGTU, 2007. - 322 p.

16. Khatnyuk I. S. Analysis of all-aluminum chip on flex (COF) technology for manufacturing hybrid microassemblies for microprocessor systems: materials of the 10th all-ukrainian scientific and technical conference of students and postgraduates "Information systems and technologies". / I. S. Khatnyuk. - Odessa: ODAKH, 2010. - p. 53-55.

17. Semenets V.V. Electronic interconnection technology / V.V. Semenets, J. Kratz, I.Sh. Nevlyudov, V.A. Palagin. - Kharkiv: SMITH, 2005. - 432 p. 